\title{
Acknowledgement to Referees
}

The Editors-in-Chief extend their gratitude and appreciation to the following reviewers whose comments and criticisms have ensured the quality of the papers published in this journal.

A. Motta, Trento

W.K. Aicher, Tübingen

B. Amsden, Kingston, $\mathrm{ON}$

D.N. Angelov, Cologne

S. Arnhold, Giessen

S.F. Badylak, Pittsburgh, PA

L. Barlow, Aurora, CO

R. Behr, Göttingen

M. Bergmann, Giessen

P.L. Bollyky, Stanford, CA

P. Boukamp, Heidelberg

T. Brand, London

D. Breitkreutz, Heidelberg-Dossenheim

B. Brown, Pittsburgh, PA

R.C. Burghardt, College Station, TX

R. Busuttil, Melbourne, VIC

J.T. Butcher, Ithaca, NY

H.F. Carvalho, Campinas

S. Cavallero, Los Angeles, CA

M.R. Celio, Fribourg

C.-C. Chen, Taipei City

P. Cinelli, Zurich

M. Coathup, Stanmore

L. Correa, Sao Paulo

S. Cotofana, Albany, NY

R. David, Rostock

F. De Francesco, Naples

C. de la Cuadra-Blanco, Madrid

L. Dini, Lecce

A. Dudley, Omaha, NE

F. Eckstein, Salzburg

G. Feigl, Graz

I. Flamme, Wuppertal

J. Fraher, Cork

M. Gauster, Graz

C. Gentile, Sydney, NSW

S. Ghazanfari, Maastricht

T. Goto, Kagoshima

M.E. Grawish, Mansoura

C. Guenter, Munich

A. Hall, Edinburgh

N. Hammer, Dunedin

S. Hirano, Kyoto

Q. Huang, Fuzhou

K. Hyndman, Birmingham, AL

E. Iacono, Ozzano dell’Emilia
R. Ibanez Rosales, Mexico City

K. Izumi, Niigata

I. Izykowska, Wroclaw

G. Jenkin, Clayton, VIC

E. Jorge, Belo Horizonte

M. Kabiri, Tehran

M. Kasper, Dresden

C. Kemmis, Winston-Salem, NC

G. Khang, Cheongju

K. Klisch, Zurich

L. Kokai, Pittsburgh, PA

B. Kruithof, Leiden

W. Kummer, Giessen

A. Kurtz, Berlin

B. Kurz, Kiel

M. Li, Beijing

N. Lindenblatt, Zurich

V. Macchi, Padova

J. Männer, Göttingen

S. Medler, Fredonia, NY

J. Meseguer Ripolles, Edinburgh

K. Messlinger, Erlangen

A. Miething, Bonn

M. Mogl, Berlin

I. Moll, Hamburg

O. Mora, Querétaro

S. Morelli, Rende

J. Morris-Wiman, Lewisburg, WV

R. Munoz-Chapuli, Malaga

W.L. Neuhuber, Erlangen

J. Nio-Kobayashi, Sapporo

A. Nohe, Newark, NJ

L. Novikov, Umeå

J.P. O’Connor, Newark, NJ

F. O'Brien, Dublin

Y. Ogata, Matsudo

I. Okic Djordjevic, Belgrade

O.N. Oliveira, Sao Carlos

K. Oprych, London

M. Paine, Los Angeles, CA

M. Pei, Morgantown, WV

M. Pretterklieber, Vienna

J. Prochazka, Vestec

M. Prost, Maastricht

G. Ramírez, Lleida

A. Ratcliffe, Del Mar, CA 
K. Rezende, São Paulo

F. Roemer, Erlangen

G.S. Roh, Jinju

J. Rohwedel, Lübeck

P. Rosset, Tours

A. Saber, Cairo

B. Sacchetti, Rome

S. Salerno, Rende

F. Sant'Ana, Brasilia

T. Sasanami, Shizuoka

R.K. Sayed, Sohag

F. Schrödl, Salzburg

G.G. Schulze-Tanzil, Nuremberg

J. Seeger, Leipzig

F. Sharifpanah, Giessen

F. Sinowatz, Munich
L. Sperling, Porto Alegre

E. Tanaka, Tokushima

M.R. Tatara, Lublin

M. Tatullo, Crotone

R. Terayama, Okayama

R. Tian, Shanghai

A. Traweger, Salzburg

R.J.-F. Tsai, Taipei

N. Tsikolia, Göttingen

F.E. Weber, Zurich

J. Wistuba, Münster

H. Witte, Ilmenau

J. Wörl, Erlangen

K. Zimmermann, Erlangen

N. zur Nieden, Riverside, CA

T.G. Zybina, St.-Petersburg 